\title{
OMAE2010-20421
}

\section{CHARACTERIZING DANGEROUS WAVES FOR OCEAN WAVE ENERGY CONVERTER SURVIVABILITY}

\author{
Justin Hovland \\ Mechanical Engineering \\ Oregon State University \\ United States
}

\author{
Robert Paasch \\ Mechanical Engineering \\ Oregon State University \\ United States
}

\author{
Merrick Haller \\ Civil Engineering \\ Oregon State University \\ United States
}

\begin{abstract}
Ocean Wave Energy Converters (OWECs) operating on the water surface are subject to storms and other extreme events. In particular, high and steep waves, especially breaking waves, are likely the most dangerous to OWECs. A method for quantifying the breaking severity of waves is presented and applied to wave data from Coastal Data Information Program station 139. The data are wave height and length statistics found by conducting a zero-crossing analysis of time-series wave elevation records. Data from two of the most severe storms in the data set were analyzed. In order to estimate the breaking severity, two different steepness-based breaking criteria were utilized, one being the steepness where waves begin to show a tendency to break, the other the steepness above which waves are expected to break. Breaking severity is assigned as a fuzzy membership function between the two conditions. The distribution of breaking severity is found to be exponential. It is shown that the highest waves are not necessarily the most dangerous. Even so, waves expected to be breaking are observed being up to 17 meters tall at station 139 .
\end{abstract}

Keywords: wave energy, survivability, breaking waves, joint distribution, OWEC

\section{INTRODUCTION}

There is currently worldwide interest in developing marine renewable energies, including wave, tidal, and thermal gradient technologies. This paper is focused on Ocean Wave Energy Converters (OWECs) and the need to accurately describe their operating environment with regards to survival. Potential OWEC designs are numerous, while several are beginning to be tested on a large scale (see [1] for a discussion of concepts, [2] for the current state of the field). However, survivability at sea is still an area of uncertainty.

Current guidelines from the European Marine Energy Centre give an extensive listing of potential factors affecting survivability, listing the "threat of storms" among them [3].
Storms produce not only high waves, but also steep and breaking waves. Much work has gone into how to specify the return period of an extreme wave height (e.g. the 100-year design wave). This is relevant for many platforms, but it is not necessarily applicable to smaller structures on the ocean surface. For example, a ship may travel over a very high yet long wave with no trouble while the same wave inundates a platform causing extensive damage. Conversely, a shorter plunging breaker may capsize the ship, but pass uneventfully under the platform. Indeed, breaking waves present a special threat to ocean-going vessels, owing largely to their slamming effect, and are responsible for damaging and capsizing many vessels [4-6]. Today, many OWECs are designed to operate on the ocean surface (as opposed to on a platform), offshore, in waters of moderate depth. In the future they may be farther offshore and in deeper waters. This puts them at the mercy of the weather on the open ocean and may also make them difficult to reach for regular maintenance and inspection. Consequently, in order to be cost effective, OWECs must not only be reliable, but survivable as well [7].

This paper summarizes an attempt at describing the breaking severity of deep water waves during storms. The method involves finding the joint distribution of wave height and period during a storm, and using breaking criteria to determine the danger presented by each wave, assuming danger is indeed linked to the breaking severity. In general, there are three ways to obtain the joint distribution: 1) theoretically, 2) by linear superposition of spectral wave components, and 3) by direct analysis of time-series data. The work presented in this paper was done using the third method, that is, using actual time-series wave elevation data. The distribution of the breaking severities was found to match an exponential distribution very well. However, this work provides no correlation between the breaking severity factor and the actual expected breaking wave geometry, which must be known in order to make an accurate estimate of the forces induced by breaking waves on OWECs and other structures. 


\section{METHODS AND DATA}

This work has been done using data from the Coastal Data Information Program (CDIP) station 139 off the coast of Oregon ( $43^{\circ} 46.00 \mathrm{~N} 124^{\circ} 33.05 \mathrm{~W} ., 186 \mathrm{~m}$ depth). The station is a Datawell directional buoy. In addition to spectral and parameter data, CDIP makes the raw time-series data available as well. The time-series data gives $\mathrm{x}$ (north-south), $\mathrm{y}$ (east-west), and $\mathrm{z}$ (vertical) displacements at $1.28 \mathrm{~Hz}$. The buoy was deployed in July 2006, so the available data set at the time of writing spans July 2006 through January 2010. While only the vertical displacement from the $x-y-z$ data was used in this work, it should be noted that the buoy is moving in a Lagrangian reference frame, that is, the buoy may have a slightly different $x-y$ location at each end of a wave. Thus, the measured wave periods may not be the same as if they had been measured in a Eulerian reference frame [8] (e.g. by a wave staff on a fixed platform).

Spectral analyses are performed by CDIP every half hour, thus sea state parameters are given in half-hour intervals. Given that $m_{0}$ is the zeroth moment of the wave energy density spectrum, the significant wave height $\left(\mathrm{H}_{\mathrm{S}}\right)$ is recorded as

$$
H_{S}=4 \sqrt{m_{0}}
$$

\section{Identifying Storms}

Dangerous sea states occur during storms, when sustained high winds generate high and steep waves. Other factors such as currents and wave directionality are not considered here. Storm events may be identified from the significant height records using a method described by Boccotti [9]. An interval in the significant height record is considered a storm if $\mathrm{H}_{\mathrm{s}}$ is above the storm threshold for more than 12 hours and does not fall below the threshold for more than 12 hours. The suggested threshold is 1.5 times the annual average $\mathrm{H}_{\mathrm{S}}(3.5 \mathrm{~m}$ for this data set). However, this value tended to yield drawn out storm events with poor distinction between peak values. A threshold value of $4 \mathrm{~m}$ was found to give a much clearer distinction between individual storm events. There were a total of 52 storm events identified with peak significant heights ranging from 4.9 to $10.4 \mathrm{~m}$. The top ten storms, ordered by the maximum significant height that occurred during the storm, are shown in Table 1. Note that there does not appear to be a direct correlation between storm duration and related intensity. This is also apparent through the Equivalent Triangular Storm concept shown by Arena and Pavone [10]. There were no storm events between April and September, and the worst storms tended to be in December and January.

Storms 1 and 7 will be discussed more throughout the paper. They are similar in shape as seen on the $\mathrm{H}_{\mathrm{S}}$ records in Figure 1, but have an interesting difference in the occurrence of dangerous waves. Specifically, it will be shown that during storm 1, steeper waves are generated at the beginning of the storm while the sea state is growing. On the other hand, steeper waves during storm 7 occur while the significant height is relatively low. This illustrates the point that the highest individual waves are not necessarily the most dangerous; wave steepness and breaking must also be accounted for.
Table 1. Top ten storms at CDIP Station 139, ranked by maximum significant wave height

\begin{tabular}{|c|c|c|c|}
\hline Rank & Start Date & Duration $(\mathbf{h r s})$ & Max $_{\mathbf{s}}(\mathbf{m})$ \\
\hline 1 & $12 / 2 / 07$ & 84 & 10.38 \\
\hline 2 & $1 / 3 / 08$ & 135 & 9.40 \\
\hline 3 & $11 / 12 / 06$ & 39 & 9.39 \\
\hline 4 & $11 / 16 / 09$ & 168 & 8.88 \\
\hline 5 & $12 / 11 / 06$ & 128 & 8.69 \\
\hline 6 & $12 / 13 / 08$ & 56 & 8.39 \\
\hline 7 & $12 / 25 / 08$ & 59 & 8.19 \\
\hline 8 & $11 / 12 / 07$ & 31 & 8.03 \\
\hline 9 & $1 / 28 / 08$ & 119 & 7.74 \\
\hline 10 & $12 / 20 / 06$ & 72 & 7.68 \\
\hline
\end{tabular}
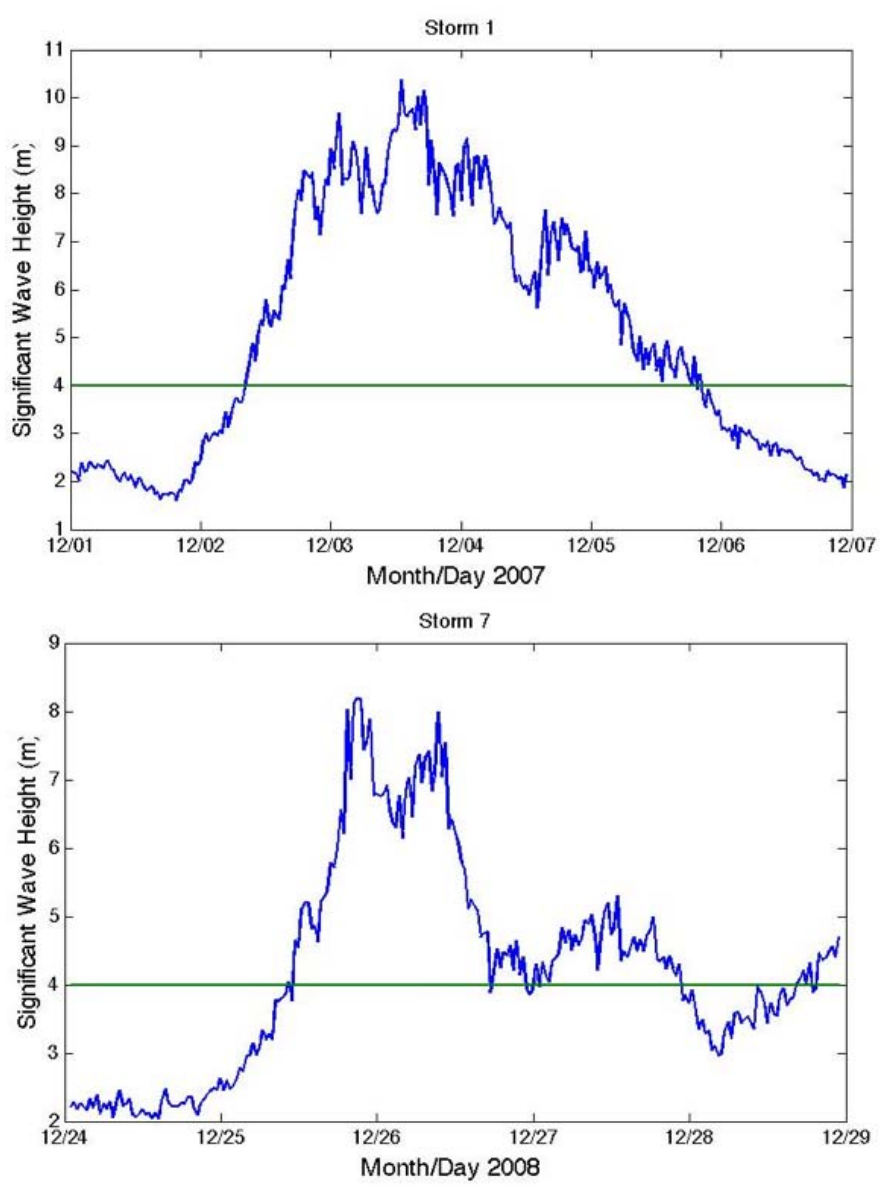

Figure 1. Significant height records of storms 1 (top) and 7 (bottom) at CDIP station 139

\section{Wave Elevation Time-series Analysis}

Once a storm interval was known, a time-series record of surface elevation during that interval was used to analyze the recorded individual storm waves. The surface elevation was analyzed using a zero down-cross technique as described by Myrhaug and Kjeldsen [11]. A wave was defined between the two points where the water surface crosses the still water line (SWL) as shown in Figure 2. The wave length $(L)$ was calculated using the deep water dispersion relation and wave period $(T)$, and the steepness $(S)$ was taken as the height over the length. 


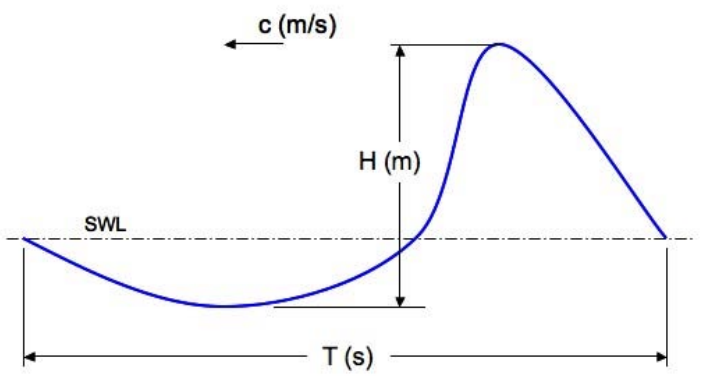

Figure 2. Zero down-cross wave definition

$$
\begin{aligned}
& L=g T^{2} / 2 \pi \\
& S=H / L
\end{aligned}
$$

Since the data is discrete and the sampling frequency was not very high, the zero crossing location was not always very well defined. Therefore, linear interpolation between the two points on either side of the SWL was used to find a more precise zero crossing point.

The buoy data is quality controlled by CDIP before it is written to the $x-y-z$ displacement file. Details of their procedures and file types can be found on the CDIP website. Data identified as 'bad' is left out of the $x-y-z$ files. Even so, some spikes are still present in the files and are generally attributed to data transmission errors. Such a spike in the wave elevation data is shown in Figure 3. Since these file errors would cause errors in the zero-crossing analysis, the data was quality controlled again in two ways. First, if a gap was found in the timestamp, then the data does not form a continuous wave and the data between the zero-crossing on either side of the gap was discarded. Second, if a spike was identified, the data between zero-crossings was discarded. Spikes were identified by a positive excursion above $10 \mathrm{~m}$, a value that was found to work well for this data set. In total, less than $1 \%$ of waves were discarded from most storms, storm 1 being a notable exception with $8 \%$ discarded, with the majority of the errors being from data gaps.

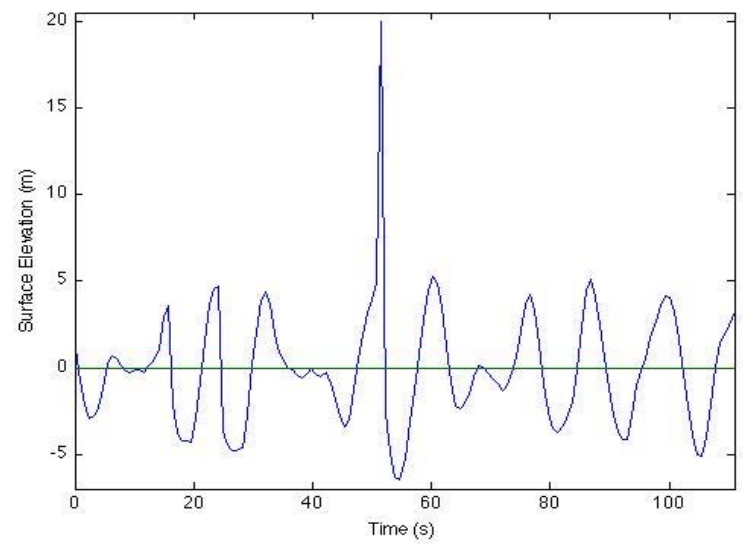

Figure 3. A spike in the wave elevation data

\section{WAVE BREAKING AND SEVERITY}

Before discussing the causes of wave breaking on deep water, it may be best to discuss why breaking waves should be considered dangerous. A spilling breaker will have a turbulent flume of water preceding its crest, while a plunging breaker can generate a vertical wall of water or a jet of water pounding down from above. Exactly how the water strikes an object on the surface will depend on the geometry of the object. In either case, the object will be subjected to impact and turbulence. The effects of such impacts on ships is discussed by Myrhaug and Dahl [5] and Kjeldsen [6]. The concern is that the impacts and turbulence could generate extreme loads on certain OWECs. Thus it would be useful to know how severe the breakers will be and how often they will occur.

To describe breaker occurrence and severity, we must have some understanding of wave breaking. In deep water, a steepness-based breaking criteria is typically assumed, though its value does not seem altogether concrete. Peirson and Banner [12] note that there are generally three ways to induce wave breaking: 1) relative motion of subsurface obstacles, 2) modulated wave train group structure, and 3) wind. Moving subsurface objects are not relevant here, and wind is generally responsible for creating microscale breakers $(\mathrm{L}<0.5 \mathrm{~m})$ that are also not relevant here. Thus, it is noted that steep and breaking waves generally arise due to wave group modulation. By this it is meant that waves at the dominant wave frequency (carrier waves) are affected by waves at other frequencies, creating a modulated wave train that is inherently unstable.

Several interesting studies have been done in connection to finding a useful breaking criterion. In the literature, steepness is often given as $k a$, where $k=2 \pi / L$ is the wave number and $a$ is the wave amplitude. Ochi and Tsai [13] found $k a=0.29$ (or $S=0.092$ ) to be accurate as a breaking criterion for irregular waves. Reid [14] observed that modulated wave packets with a steepness greater then $a k=$ $0.20(S=0.064)$ will evolve to breaking. Melville [15] observed a similar situation, noting that waves with a higher initial steepness would break with greater intensity. These two values may be used as upper and lower breaking criteria to estimate the severity with which a given wave may break. If we take the lower value as zero, and the upper value as unity, then we may define the breaking severity as a function of steepness:

$$
\begin{gathered}
f(S)=\left\{\begin{array}{cl}
0 & \text { for } S_{N} \leq b \\
\left(S_{N}-b\right) /(1-b) & \text { for } b<S_{N}>1 \\
1 & \text { for } S_{N} \geq 1
\end{array}\right. \\
S_{N}=S / 0.092 \\
b=0.064 / 0.092=0.69
\end{gathered}
$$

where $S_{N}$ is the wave steepness normalized to the upper breaking criteria, and $b$ is the normalized value of the lower breaking criteria. This is in essence a fuzzy membership function, and is shown more clearly in Figure 4. 


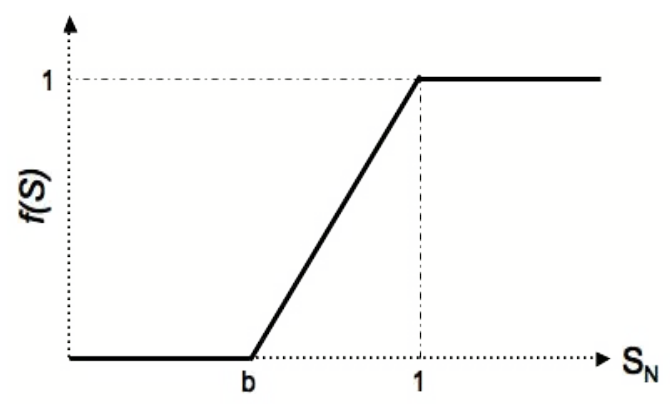

Figure 4. Fuzzy membership function for wave steepness

\section{A Breaking Severity Factor}

The danger presented by a breaking wave will not depend only on how steep the wave is, but will also depend on its size. Thus, it would be useful to have a factor that described both the height and steepness of a wave. Given a function of wave height, $f(H)$, we may define a breaking severity factor as

$$
B=f(H) f(S)
$$

Lacking a single sensible value to normalize the wave height against, the function $f(H)$ may simply be taken as $H$. Then (7) becomes

$$
B=H \cdot f(S)
$$

Note that this parameter has units of length. It is possible that this parameter may be related to some physical dimension of breaking waves, such as the likely height of the breaking front, or possibly the distance for which it will break. If such a relation exists, a calibrating coefficient would need to be introduced to properly adjust the numerical values, and such a coefficient would need to be found experimentally. At this time no such relation exists and this paper does not attempt to introduce one. It will suffice for now to remember that given a breaking severity factor, it means that a wave at least as high as the factor is expected to be breaking. For example, a factor of 12 means that a 12-meter wave is "fully breaking", or a higher wave is "partially breaking".

\section{JOINT DISTRIBUTION OF HEIGHT AND PERIOD}

Viewing waves on a joint distribution plot allows us to see how all of the waves are related in both height and period. However, it lacks a time dimension, so it is difficult to see how the wave field evolves over time. In particular, it may be of interest to compare wave steepness at different times in a storm. To show this, we may plot the joint distribution of wave height and period for different intervals during a storm. For example, we can split a storm into three intervals: beginning, middle, and end. Figures 5 and 6 show the joint distribution of wave height and period during storms 1 and 5, respectively. The upper $(S=0.092)$ and lower $(S=0.064)$ breaking conditions are shown as well.

From Figure 5 it is apparent that the waves are generally high for the entirety of storm 1 , but have relatively short periods at the beginning that become longer as the storm progresses. Thus the steepest and potentially most dangerous waves occur earlier in the storm.
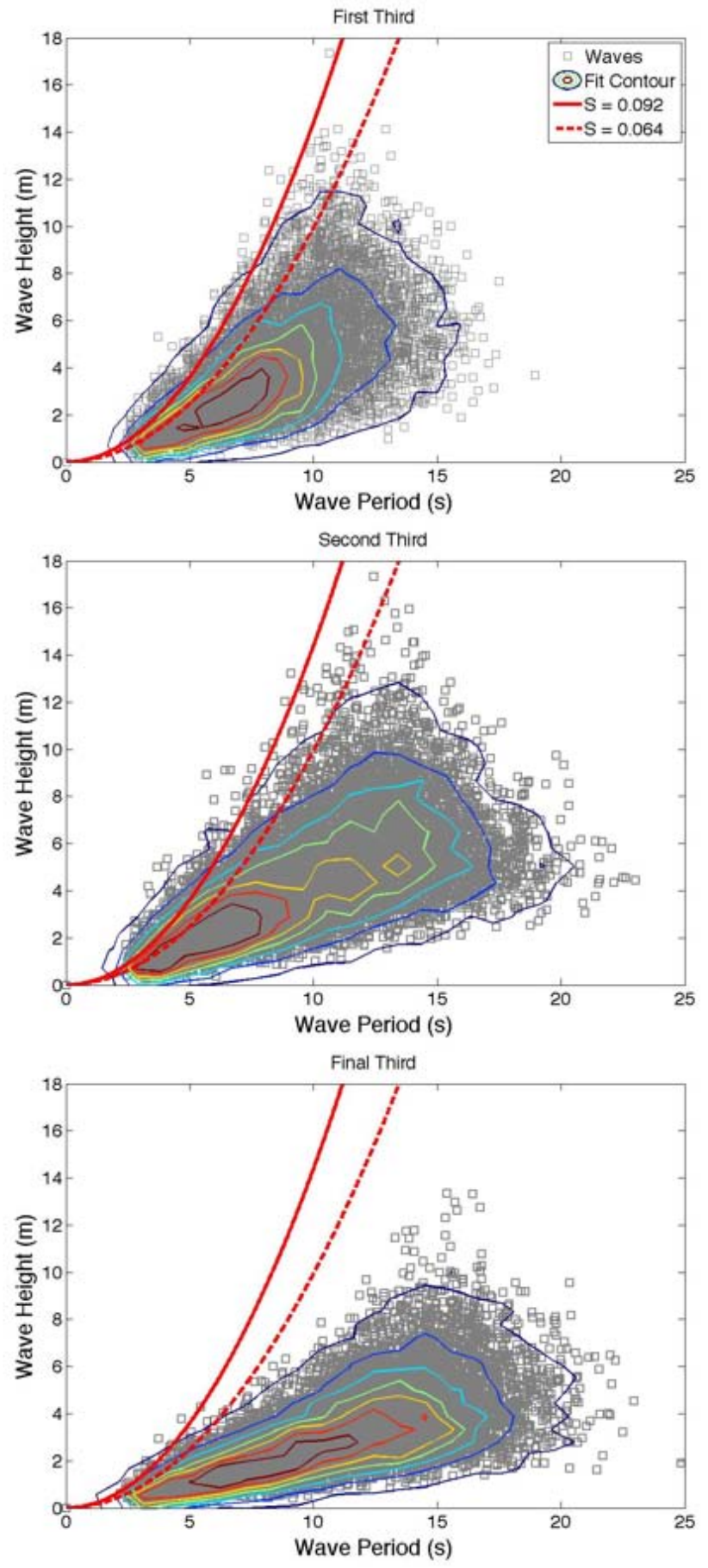

Figure 5. Joint distribution of wave height and period during storm 1

Figure 6 shows the opposite trend for storm 7. While the waves are higher during the first part of the storm, they are not generally in the breaking region. During the final third of the storm however, there is a high concentration of steep waves. Referring back to Figure 1, we see that the significant wave height during the final third of the storm is not very high ( $4.5 \mathrm{~m}$ ), yet this is where the most dangerous waves of the storm seem to be occurring.

The steepest edge of all of the distributions shown seems to follow a line of constant steepness (particularly the first third of storm 1 and the final third of storm 7), indicating that 
the waves are indeed subject to a steepness limit. However, some of the waves are seen to be above the upper breaking condition. In this analysis, these waves are simply assigned $f(S)=1$, and their breaking severity factor is equal to their height. This raises some question on the adequacy of these breaking conditions, but a greater question is the effect that breaking waves have on the wave record. If water overtakes the recording buoy, then the buoy will be subject to more chaotic motion and the record will not reflect the true water surface. This process may be why so many short 'waves' are on record which surpass the breaking condition.
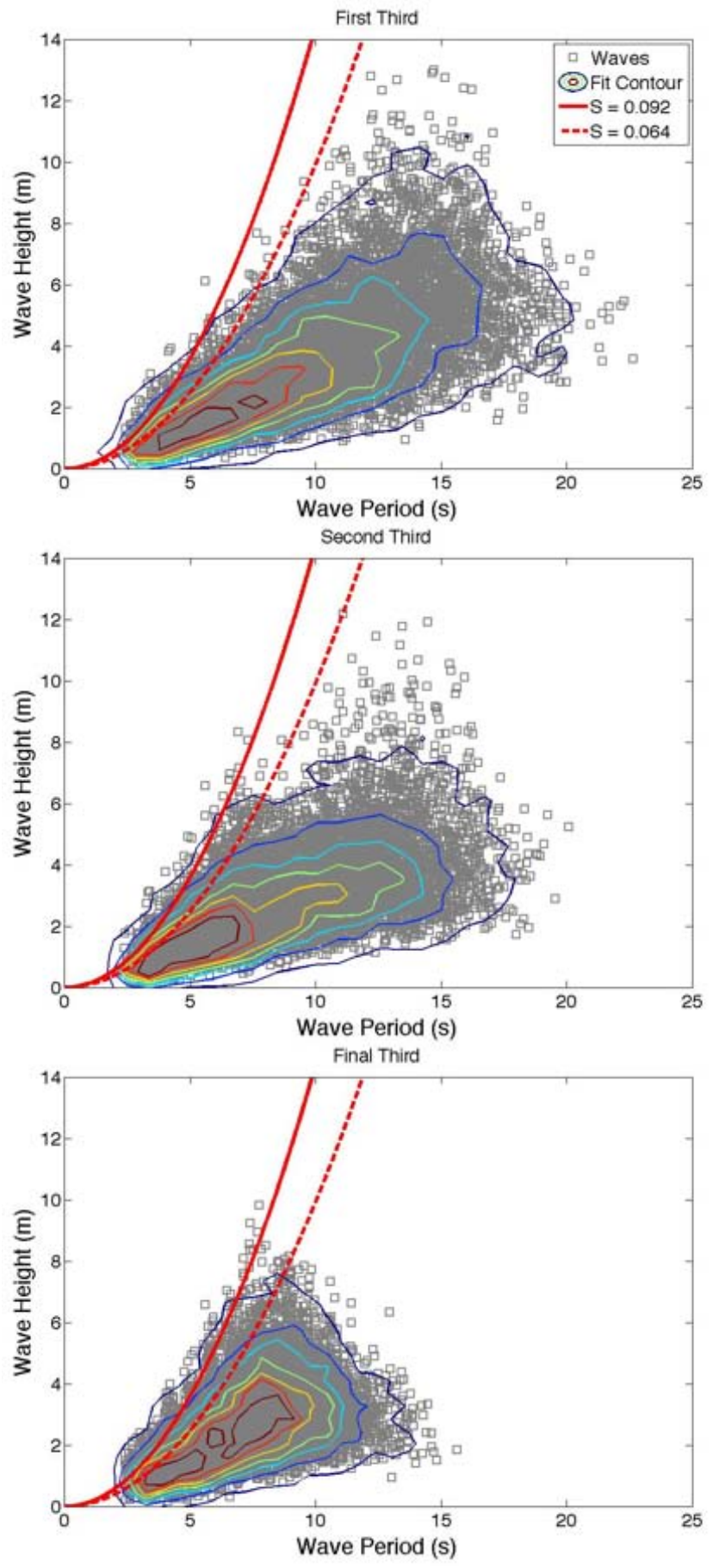

Figure 6. Joint distribution of wave height and period during storm 7

\section{BREAKING SEVERITY FACTOR DISTRIBUTION}

The breaking severity factors calculated using (8) are plotted on the next page in Figure 7 for storms 1 and 7 . The wave height is also plotted for each storm. The horizontal axis on these plots is incremented by wave. That is, every point on the horizontal axis represents one zero-crossing wave during the storm. The first wave of the storm is the first point on the left, the last wave of the storm is the last point on the right. There are almost 30,000 waves in each storm, so each plot contains that many points. Note that on the breaking severity factor plots most of the points are zero, because only waves with $S>0.064$ have a breaking severity factor. The purpose of these plots is to illustrate the fact that high waves do not necessarily mean dangerous waves. During storm 1 we see that there are four intervals when the waves are noticeably higher. In contrast, the breaking severity factor drops off steadily during the second half of the storm, and the subsequent resurgences of high waves do not produce a corresponding increase in breaking severity. However, it is true for storm 1 that the intervals of highest breaking severity factors roughly correspond to intervals of high waves. During storm 7 we notice that the waves are high in the first half of the storm, and are generally lower in the second half, while the breaking severity factor is low for the first half and high during the second half.

\section{Cumulative Probability Distribution}

Since we want to know about how breaking waves are distributed in a storm and how often they will occur, it is useful to plot the breaking severity factor against its cumulative probability. That is, given $n$ breaking waves in a storm (those above the lower threshold), the probability of the most dangerous wave is $1 / n$. Such a plot was found to be well fitted by the exponential distribution

$$
P(X \geq B)=e^{-\lambda B}
$$

where $P(X \geq B)$ means the probability of the set of breaking waves with a breaking factor greater than or equal to $B$. The parameter $\lambda$ indicates how quickly the probability of dangerous waves falls. Given a number of breaking waves, a lower value of $\lambda$ indicates that more dangerous waves are probable. This distribution is plotted for storms 1 and 7 in Figure 8 using a logarithmic scale on the ordinate axis. This plot emphasizes the more dangerous, lower probability waves, and $\lambda$ is apparent as the slope of each line.

The breaking factor for the most dangerous wave of a given storm may be calculated by setting the RHS of (9) equal to $1 / n$ and solving for $B$ :

$$
B_{\max }=\frac{1}{\lambda} \ln (n)
$$

Table 2 lists the parameter $\lambda$ along with the actual and calculated maximum breaking severity factor for the top ten storms introduced in Table 1 . The calculated maximum breaking severity factor was found using (10), where $n$ was the number of breaking waves. 

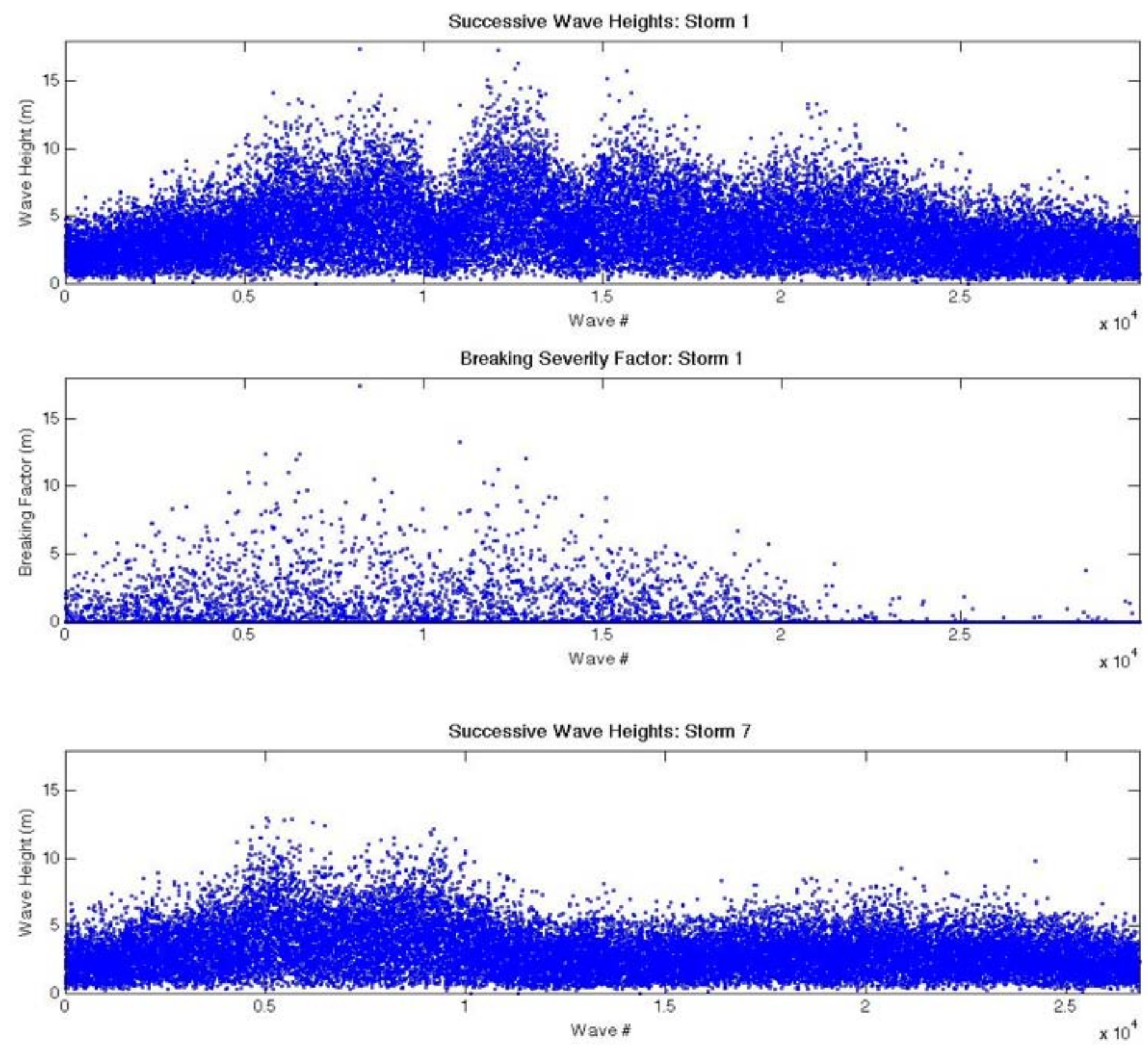

Breaking Severity Factor: Storm 7

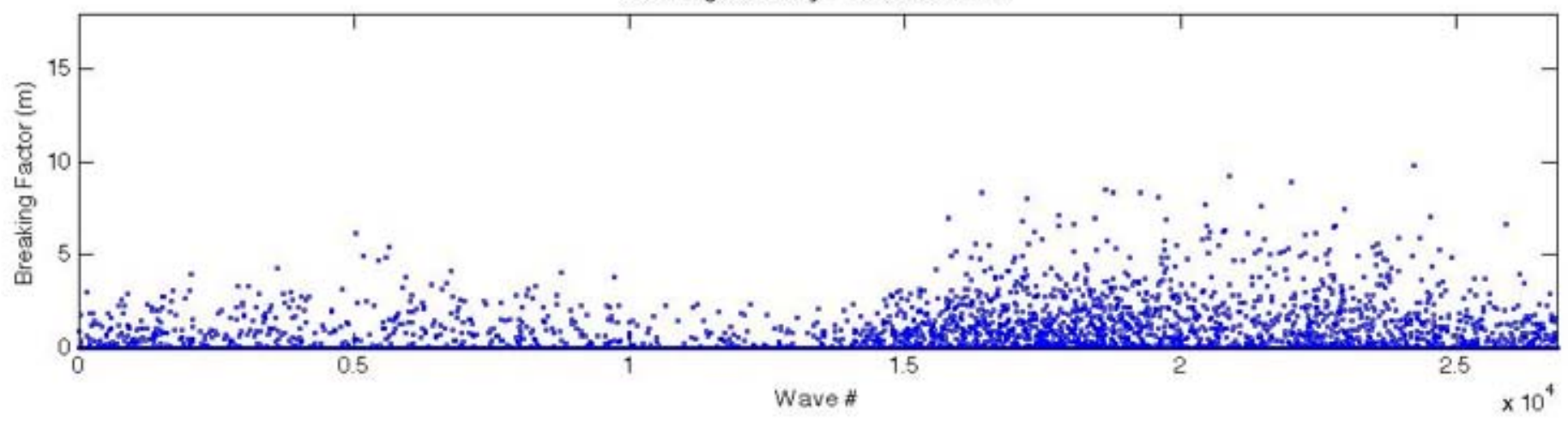

Figure 7. Wave heights and breaking severity factors for storm 1 (top) and storm 7 (bottom) 


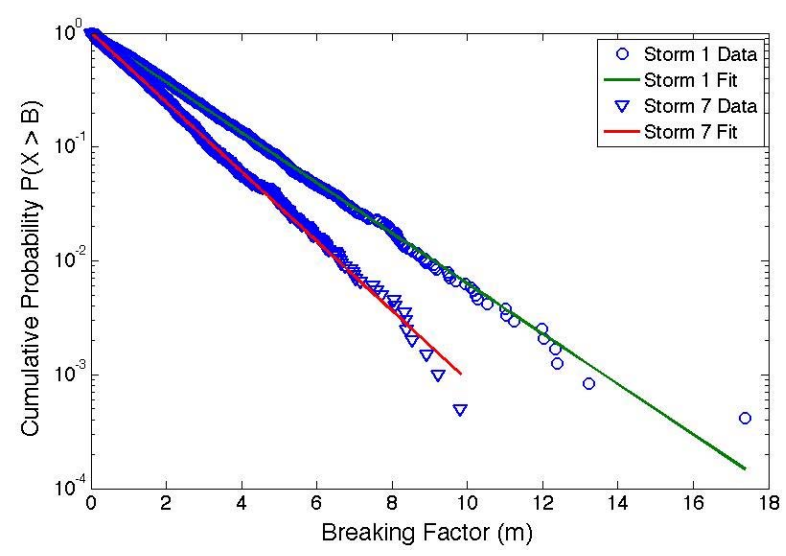

Figure 8. Cumulative distribution of the breaking factor during storm 1 (upper) and storm 7 (lower)

Table 2. Breaking severity factor distribution parameters

\begin{tabular}{|c|c|c|c|c|c|}
\hline $\begin{array}{c}\mathbf{H}_{\mathbf{s}} \\
\text { Rank }\end{array}$ & $\begin{array}{c}\text { Total } \\
\text { Waves }\end{array}$ & $\boldsymbol{n}$ & $\boldsymbol{\lambda}$ & $\begin{array}{c}\text { Observed } \\
\mathbf{B}_{\max }(\mathbf{m})\end{array}$ & $\begin{array}{c}\text { Calculated } \\
\mathbf{B}_{\max }(\mathbf{m})\end{array}$ \\
\hline 1 & 29982 & 2393 & 0.509 & 17.37 & 15.3 \\
\hline 2 & 38075 & 2534 & 0.774 & 11.05 & 10.1 \\
\hline 3 & 13275 & 799 & 0.464 & 18.92 & 14.4 \\
\hline 4 & 70755 & 5051 & 0.679 & 11.69 & 12.6 \\
\hline 5 & 35376 & 2611 & 0.531 & 14.75 & 14.8 \\
\hline 6 & 19151 & 935 & 0.773 & 9.24 & 8.8 \\
\hline 7 & 26829 & 2006 & 0.703 & 9.82 & 10.8 \\
\hline 8 & 11958 & 591 & 0.550 & 11.92 & 11.6 \\
\hline 9 & 51532 & 3389 & 0.819 & 9.07 & 9.9 \\
\hline 10 & 27157 & 1513 & 0.660 & 12.32 & 11.1 \\
\hline
\end{tabular}

\section{Implications for OWEC Design}

In order to determine the forces a breaking wave will induce on a structure the geometry of the wave must be given. Assuming a geometry, an approximate slamming load may be calculated (see Chakrabarti [16]). Finally, the response of the structure may be predicted and used to aid in the design process. Examples of structural response analyses have been given by Boccotti [9], primarily for breakwaters, and by Chakrabarti [16] for fixed and floating offshore platforms. These well-known procedures should be of great use to any OWEC designer.

An issue that needs further clarification is exactly how the breaking severity factor correlates to the actual breaking wave geometry. A higher breaking severity factor only states that a wave of at least a given height is expected to be breaking 'more severely'. While this does not yet help elucidate the force distribution a structure will be subjected to, it may still be useful for a qualitative discussion on the repercussions of breaking waves on structure geometry or site selection.

The possibility of high breaking waves brings up the question of OWEC survivability. No matter the device efficiency, it will be useless if it cannot survive storms at sea. The vulnerability and survival strategies of OWECs do not seem to have been rigorously addressed to date, but there seems to be three general ways to handle dangerous waves.
First, all vulnerable parts can be located safely above the water surface as is done on most platforms. This seems impractical for OWECs because they need to operate at the water surface and constructing such platforms would likely be prohibitively expensive. Second, the body and structure of the OWEC must be designed to directly withstand the force of severe breaking waves. To achieve this with confidence, the distribution of expected wave geometries must be known. Finally, the device could be located below the water surface where wave breaking would have little effect. If the device is submerged only when threatened, then the designer should know how often a given site will produce a sufficient threat. Knowing the breaking severity distribution at the site could help evaluate the threat.

\section{CONLCUSIONS}

A breaking severity factor for deep water waves was defined as the wave height times a function of its steepness. Two steepness based breaking criteria were utilized to define the function. All of the waves in two separate storms were presented on a series of joint distribution plots which also show the breaking criteria. Each wave was identified by applying a zero down-crossing analysis to actual wave elevation time-series data. The breaking severity factor of each wave was also shown for both storms, and the cumulative distribution of the breaking severity factor was shown to be well fitted by an exponential distribution. Using this distribution, OWEC designers can predict how many breaking events of a given severity a device will be subjected to during a storm. This knowledge can assist in the effort to increase the survivability of OWECs by guiding the improvement of OWEC designs, and by identifying risks at a given site.

\section{ACKNOWLEDMENTS}

I would like to thank my co-authors, Dr. Paasch and Dr. Haller for helping me develop my work and paper. Thanks to Pukha Lenee-Blum, Kelley Ruehl, and Steve Meicke for their help in editing. Special thanks to Richard Frels for his detailed knowledge about the behavior and dangers of the sea.

This work was completed under the auspices of the Northwest National Marine Renewable Energy Center (NNMREC), funded by the US Dept. of Energy. 


\section{REFERENCES}

[1] M.E. McCormick, Ocean Wave Energy Conversion, Dover Publications, 2007.

[2] J. Cruz, Ocean Wave Energy: Current Status and Future Perspectives, Springer, 2008.

[3] European Marine Energy Centre, "Guidelines for Reliability, Maintainability and Survivability of Marine Energy Conversion Systems," 2009.

[4] C.B. Smith, Extreme Waves, Joseph Henry Press, 2006.

[5] D. Myrhaug and E. Dahle, "Ship Capsize in Breaking Waves," Fluid Structure Interaction in Offshore Engineering, Computational Mechanics Publ, 1994, p. 43.

[6] S. Kjeldsen, "Measurements of Freak Waves in Norway and Related Ship Accidents," RINA, Royal Institution of Naval Architects International Conference - Design and Operation for Abnormal Conditions III, January 26, 2005 - January 27, 2005, London, United kingdom: Royal Institution of Naval Architects, 2005, pp. 31-38.

[7] A. Brown, "Towards Reliable and Survivable Ocean Wave Energy Converters," Masters Thesis, Oregon State University, 2009.

[8] M. Longuet-Higgins, "Eulerian and Lagrangian Aspects of Surface Waves," Journal of Fluid Mechanics, vol. 173, 1986, pp. 683-707.

[9] P. Boccotti, Wave Mechanics for Ocean Engineering, Elsevier Science, 2000.

[10] F. Arena and D. Pavone, "Some Statistical Properties of Random Waves in a Sea Storm," 25TH International Conference on Offshore Mechanics and Arctic Engineering, OMAE 2006, June 4, 2006 - June 9, 2006, Hamburg, Germany: ASME, 2006.

[11] D. Myrhaug and S.P. Kjeldsen, "Steepness and Asymmetry of Extreme Waves and the Highest Waves in Deep Water," Ocean Engineering, vol. 13, 1986, pp. 549-568.

[12] W.L. Peirson and M.L. Banner, "On the Strength of Sreaking of Deep Water Waves," Coastal Engineering 2000 - 27th International Conference on Coastal Engineering, ICCE 2000, July 16, 2000 - July 21, 2000, Sydney, NSW, Australia: American Society of Civil Engineers, 2004, pp. 369-381.

[13] M.K. Ochi and C. Tsai, "Prediction of Occurrence of Breaking Waves in Deep Water," Journal of Physical Oceanography, vol. 13, 1983, pp. 2008-2019.

[14] J. Reid, "The Sideband Instability and the Onset of Wave Breaking," Breaking Waves: IUTAM Symposium, Sydney, NSW, Australia: Springer, 1991, pp. 155 - 159.

[15] W. Melville, "Wave Modulation and Breakdown," Journal of Fluid Mechanics, vol. 128, 1983, pp. 489506.

[16] S. Chakrabarti, Handbook of Offshore Engineering: Volume 1, Elsevier Science, 2005. 